\title{
Electrorheological Performance of Chitosan Phosphate Suspension
}

\author{
Ung-Su CHOI, Young-Gun Ko, and Jae-Yong KIM* \\ Tribology Center, Korea Institute of Science and Technology, \\ P. O. Box 131, Cheongryang, Seoul, Korea \\ *Dept. of Environ. Eng. Chungbuk Nat'l Univ., Cheong-ju, Korea
}

(Received November 5, 1999)

\begin{abstract}
The electrorheological (ER) performance of chitosan phosphate suspension in silicone oil was investigated. Chitosan phosphate suspension showed a typical ER response (Bingham flow behavior) and exhibited an excellent shear yield stress due to the formation of multiple chains upon the application of the electric field. The shear stress for chitosan phosphate suspension showed a linear dependence on the volume fraction of particles and 1.67 power of the electric field. The experimental results for the chitosan phosphate suspension correlated with the conduction model for ER response.
\end{abstract}

KEY WORDS Electrorheological Fluids / Chitosan Phosphate Suspension / Conduction Model / Bingham Flow $/$

Electrorheological (ER) fluids are smart materials which have the ability to control with electric field mechanical devices such as shock absorbers, dampers, clutchs and engine mounts. ${ }^{1,2}$ The ER behavior is characterized by a rapid and reversible increase in apparent viscosity due to the formation of particle chains upon application of the electric field. ${ }^{3-5}$ Since the ER effect was discovered by Winslow ${ }^{5}$ in the late $1940 \mathrm{~s}$, polarization models based on the point-dipole approximation,with focus on the mismatch between the real components of the dielectric permittivities of the particles and base fluid, were proposed to explain the behavior. ${ }^{6,7}$ Recently, the importance of the conductivity of base fluids which is strongly dependent on the electric field, has been demonstrated leading to the conduction model. The conduction model considers that the ER effect with a dc field is induced by the mismatch of the conductivity of the particles and the base fluid, given by the ratio of the conductivity of particles to that of the base fluid, $\Gamma_{\sigma}=\sigma_{\mathrm{p}} / \sigma_{\mathrm{f}}(0)$. The conduction model was originally proposed by Foulc et $a{ }^{8}{ }^{8}$ and modified by Davis and Ginder, ${ }^{9}$ Tang et al. ${ }^{10}$ and $\mathrm{Wu}$ and Conrad. ${ }^{11}$ In these models the conductivity of the base fluid is presented by a simplified expression for Onsager's electric field-enhanced ionic dissociation theory ${ }^{12}$

$$
\sigma_{\mathrm{f}}=\sigma_{\mathrm{f}}(0)\left[(1-A)+A \exp \left(E / E_{\mathrm{c}}\right)^{1 / 2}\right]
$$

where $E$ is the electric field, $\sigma_{\mathrm{f}}(0)$ conductivity at low field and $A$ and $E_{\mathrm{c}}$ are constants which depend on the base fluid. It gives the following experssion for the shear yield stress of ER fluids

$$
\tau_{\mathrm{E}} \propto \phi K_{\mathrm{f}} f\left(E_{0}, \Gamma_{\sigma}, A, E_{\mathrm{c}}\right)
$$

where $\phi$ is the volume fraction of particles, $K_{\mathrm{f}}$ the dielectric permittivity of the base fluid, $E_{0}$ the electric field, $\Gamma_{\sigma}$ the ratio of the conductivity of particles to that of the base fluid.

ER fluids are nonaqueous suspensions composed of electrically polarizable particles dispersed in a dielectric fluid and especially, the disperse phase plays an impor- tant role in the ER phenomenon. The organic polymers, cellulose, ${ }^{13}$ polyaniline ${ }^{14}$ and polyurethane ${ }^{15}$ as the disperse phases have been widely used for the formulation of ER fluids.

Because they have polar groups such as hydroxy $(-\mathrm{OH})$, amino $\left(-\mathrm{NH}_{2}\right)$ and amino-cyan $(-\mathrm{NHCN})$ radicals, respectively, suspensions of these particles provide ER effect upon application of the electric field. The chemical structure of the organic polymers is therefore important in the ER effect.

Chitosan as the base material is a natural organic biocompatible polymer from chitin by $N$-deacetylation and composed of poly D-glucosamine. It has been widely used for applications to the fields of biochemisry, pharmacology, emzymology, microbiology, agriculture and Environment. ${ }^{16}$ Chitosan suspension showed the ER behavior upon the application of the electric field due to the polarizability of the branched amino group. ${ }^{17} \mathrm{~A}$ chitosan derivative as the new anhydrous disperse phase, chitosan phosphate has been synthesized by phosphorylation reaction between chitosan and phosphoric acid.

This study describes the ER behavior of chitosan phosphate suspension and to investigate the possibility of a new ER fluid.

\section{EXPERIMENTAL}

\section{Materials}

The base fluid used was silicone oil provided by Dow Corning with a specific gravity of 0.97 , a kinematic viscosity of $50 \mathrm{cst}$ at $40^{\circ} \mathrm{C}$ and a dielectric constant of 2.61 at $25^{\circ} \mathrm{C}$. The chitosan used as base material was a commercial powder provided by Jae-kwang Co. (Korea) and contained nitrogen content of $4.8 \mathrm{wt} \%$ and its molecular weight was 10000 . Chitosan phosphate as the disperse phase was synthesized by phosphorylation reaction between chitosan and phosphoric acid ${ }^{18}$ and contained phosphorous content of $8.8 \mathrm{wt} \%$, and its particle size was $25 \mu \mathrm{m}$ in average diameter. Prior to blending the suspension, chitosan phosphate particles were dried for $5 \mathrm{~h}$ at $150^{\circ} \mathrm{C}$ and the silicone oil for $3 \mathrm{~h}$ at $130^{\circ} \mathrm{C}$ to remove moisture in vacuum oven. Chitosan phosphate suspen- 


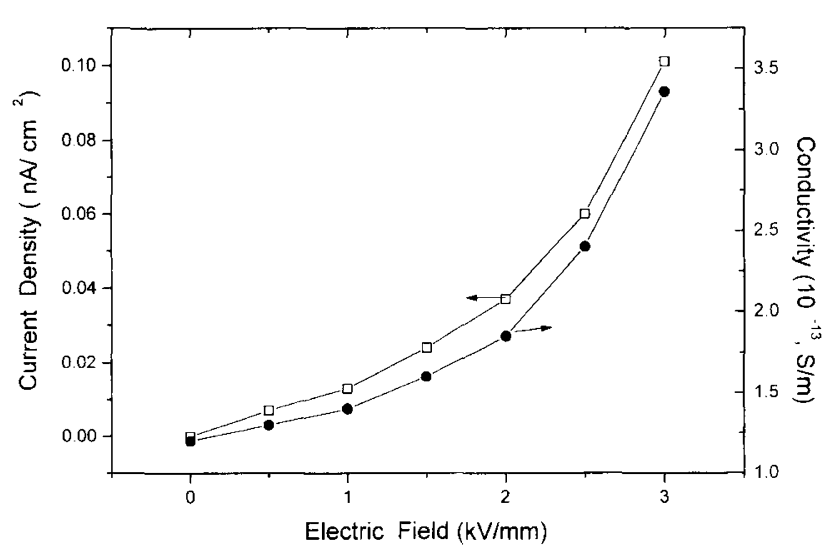

Figure 1. Effects of electric field on current density and conductivity of silicone oil.

sions were prepared at volume fractions of 0.1 to 0.4 . After vigorous mixing, the suspensions were stored in a dessicator to maintain the dry state.

\section{Electrical Tests}

The dc current density $J$ and conductivity $\sigma$ of the silicone oil and of the chitosan phosphate suspension were determined at room temperature by measuring the current passing through the fluid upon application of the electric field $E_{0}$ and dividing the current by the area of the electrodes in contact with the fluid. Current was determined from the voltages drop across a $1 \mathrm{M}$ resistor in series with the metal cell containing the oil using a voltmeter with a sensitivity of $0.01 \mathrm{mV}$. This method gave a current measuring sensitivity of $0.01 \mathrm{nA}$. DC conductivity was taken to be $\left(\sigma=J / E_{0}\right)$.

\section{Rheological Tests}

Rheological properties of the suspension were investigated in a dc field using a Physica Couette-type rheometer with a $1 \mathrm{~mm}$ gap between the bob and cup. The resistance to shear produced by the suspensions was measured as a torque on the drive shaft and then converted to shear stress and viscosity. Shear stress for the suspension was measured under shear rates of 1 to $300 \mathrm{~s}^{-1}$, electric fields of 0 to $3.0 \mathrm{kV} \mathrm{mm}^{-1}$ and volume fractions of 0.1 to 0.4 , respectively.

\section{RESULTS}

\section{Electrical Properties}

ER fluids essentially function as leaky capacitors in an ER device and the transfer of charge between particles results in an electric current through the fluid. The current density associated with a particular ER fluid is useful for estimating the power consumption of an ER device. The electrical properties of ER fluids are therefore important for predicting power requirements for the design of an ER device and also idenfying the ER mechanism. Figure 1 shows the current density and the conductivity of the silicone oil with the electric field. As seen in Figure 1, the non-Ohm character of the behavior is evident. The conductivity parameters, $\sigma_{\mathrm{f}}(0), A$ and $E_{\mathrm{c}}$ of the silicone oil were calculated using eq 1 to give $\sigma_{\mathrm{f}}(0)=$ $1.2 \times 10^{-13} \mathrm{~S} \mathrm{~m}^{-1}, A=0.0007$ and $E_{\mathrm{c}}=0.11 \mathrm{kV} \mathrm{mm}^{-1}$.

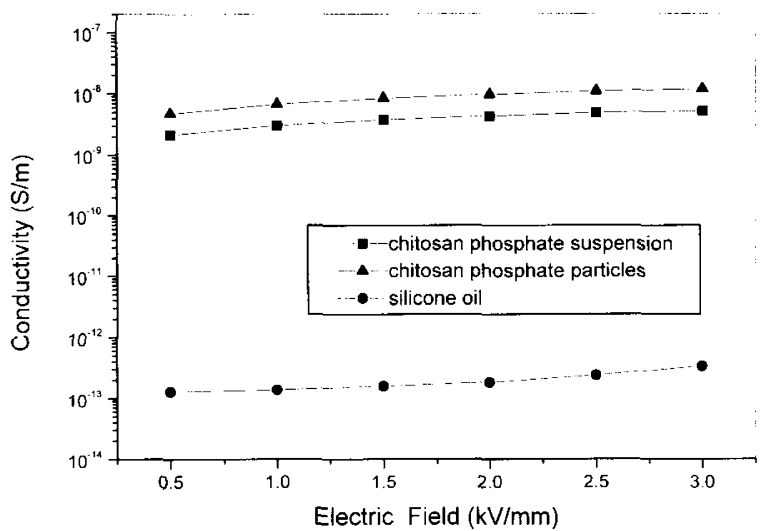

Figure 2. Effects of electric field on conductivity for chitosan phosphate suspension $(\phi=0.3)$, chitosan phosphate particles and silicone oil.

The conductivities of the chitosan phosphate suspension for a volume fraction ( $\phi=0.3$ ), chitosan phosphate particles and silicone oil $v s$. electric field are given in Figure 2. In Figure 2, the conductivity of the chitosan phosphate suspension is about 5 orders of magnitude higher than that of the silicone oil. The conductivity of the chitosan phosphate particles was calculated from the data in Figures 1 and 2 assuming that the structure consisted of single-row chains with numbers of chains per unit area

$$
N_{\mathrm{A}}=(3 / 2) \phi / \pi a^{2}: \sigma_{\mathrm{s}}=(3 / 2) \phi \sigma_{\mathrm{p}}+\sigma_{\mathrm{f}}(1-3 / 2 \phi)
$$

where $\phi$ is the volume fraction of particles. ${ }^{11}$ And the results in Figure 2 show that the conductivity of chitosan phosphate particles is about 2 times higher than that of chitosan phosphate suspension. The increase in conductivity of chitosan phosphate particles is considered to result from the polarizability of the phosphoryl radicals caused by the reaction between chitosan and phosphorous acid.

\section{Rheological Properties}

To investigate the effects of chitosan phosphate suspension on the rheological properties, studies were carried out by varying shear rates, electric fields and volume fractions.

The effect of shear rate on the shear stress for chitosan phosphate suspension is illustrated in Figure 3 . As seen in Figure 3, the chitosan phosphate suspension behaves as a Newtonian fluid without the electric field, but upon application of the electric field, it exhibits a shear yield stress $\tau_{\mathrm{E}}$, which is followed by a relatively constant shear stress. This suspension approximates a Bingham-type behavior, which is described by the equation

$$
\tau=\tau_{\mathrm{E}}(E, \dot{\gamma})+\eta \dot{\gamma}
$$

where $\eta$ is the viscosity of the suspension and $\dot{\gamma}$ the shear rate. The results in Figure 3 indicate that chitosan phosphate suspension shows excellent ER response upon application of the electric field. Figure 4 gives a log plot of $\tau_{\mathrm{E}} v s . E^{2}$ for the chitosan phosphate suspensions. 


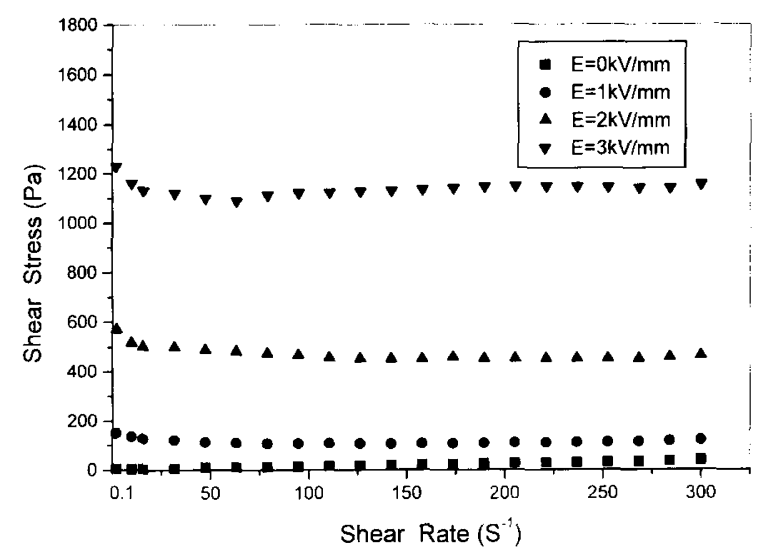

Figure 3. Shear stress $v s$. shear rate for chitosan phosphate suspension ( $\phi=0.3)$.

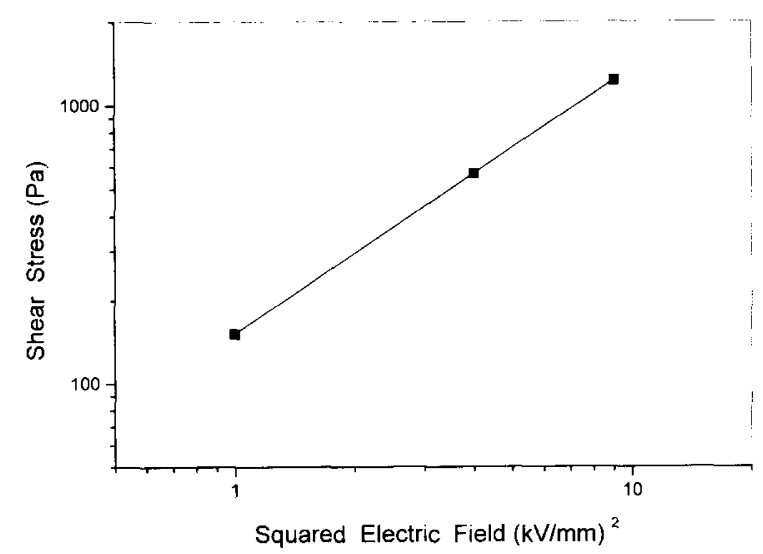

Figure 4. Effects of squared electric field on shear stress for chitosan phosphate suspension $\left(\dot{\gamma}=2 \mathrm{~s}^{-1}, \phi=0.3\right)$.

The results are for shear rate of $2 \mathrm{~s}^{-1}$ and volume fraction of 0.3 . Figure 4 indicates that the shear yield stress is proportional to 1.67 power of the electric field, i.e., $\tau_{\mathrm{E}}$ $\propto E^{1.67}$ for chitosan phosphate suspension. The effect of volume fraction of chitosan phosphate particles in the silicone oil on the shear stress is given in Figure 5. The results were obtained at a shear rate of $2 \mathrm{~s}^{-1}$. In Figure 5 , the shear stress increases in a linear fashion with the volume fraction of chitosan phosphate particles.

\section{DISCUSSION}

To explain the ER behavior of chitosan phosphate suspension, we examine the results obtained with the assumption that the base fluid and particles behave as ideal dielectric materials, and particles are aligned in chains or columns between electrodes. With these assumptions, the theological analysis of Conrad et al. ${ }^{19}$ gives for the polarization component of the yield stress

$$
\begin{aligned}
\tau_{\mathrm{E}}= & 44.1 A_{\mathrm{s}} \phi \varepsilon_{0} K_{\mathrm{f}}(\beta E)^{2} \mid\left\{\exp \left[(14.84-6.165(R / a)) \beta^{2}\right]\right\} \\
& \times\left[1 /(R / a)^{8}-4 /(R / a)^{10}\right]^{1 / 2} \mid{ }_{\max }
\end{aligned}
$$

where $A_{\mathrm{s}}$ is taken to be a structure factor pertaining to the alignment of the particles. It is equal to one for perfectly aligned single-row chains and may a value of the orders of $\sim 10$ for multiple chains or columns. $K_{\mathrm{f}}$ is the

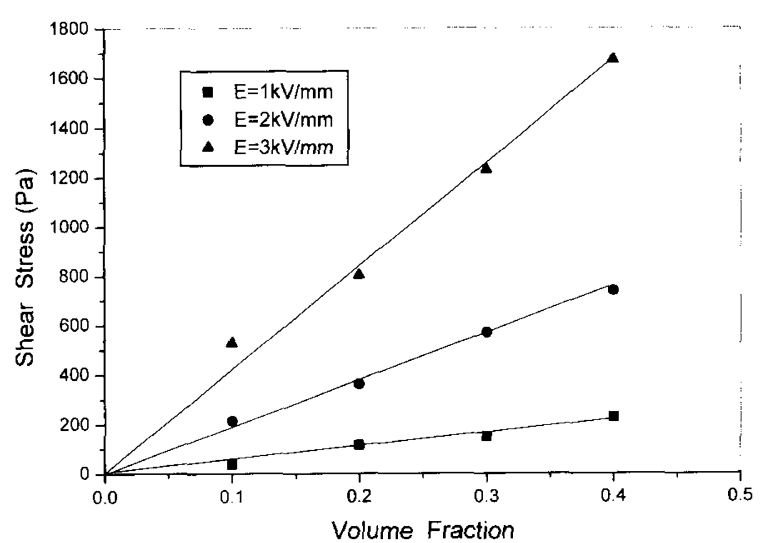

Figure 5. Effects of volume fraction on shear stress for chitosan phosphate suspension $\left(\dot{\gamma}=2 \mathrm{~s}^{-1}\right)$.

dielectric constant, $\beta$ the relative polarizability $\left[\beta=\left(\sigma_{\mathrm{p}}\right.\right.$ $\left.-\sigma_{\mathrm{f}}\right) /\left(\sigma_{\mathrm{p}}+2 \sigma_{\mathrm{f}}\right)$ under dc field, $\beta \cong 1$ ] and $R / a$ the ratio of the separation of the particles center to their radius ( $R / a \cong 2.05$ ). The structure factor, $A_{\mathrm{s}}$ is obtained from the ratio value of measured to calculated shear stress using eq 5 , that is, $A_{\mathrm{s}}=\tau_{\text {meas. }} / \tau_{\text {calc. }}$ We obtain $A_{\mathrm{s}}=3-4$ for chitosan phosphate suspension under the test conditions at a shear rate of $2 \mathrm{~s}^{-1}$, the electric fields of 1 to 3 $\mathrm{kV} \mathrm{mm}-1$ and a volume fraction of 0.3 . This may be due to the formation of multiple chains aligned between electrodes for chitosan phosphate suspension. ${ }^{19,20}$ The conduction model given by eq 2 is applied, wherein the conductivity parameters affecting the ER behavior are $\Gamma_{\sigma}$, $A, E_{\mathrm{c}}$, and $\sigma_{\mathrm{f}}(0)$. We compare the experimental values of the shear stress with those predicted by the conduction models of Tang et al..$^{10}$ and Wu and Conrad. ${ }^{11}$ The conduction model of Tang et al. gives the following expression for shear stress

$$
\tau_{\mathrm{E}}=A_{\mathrm{s}} K_{\mathrm{f}} \tau_{0} \phi E_{0}^{2}
$$

where $\tau_{0}$ is given as follows for $\Gamma_{\sigma}>10^{3}$

$$
\tau_{0}=m_{1}\left(E_{\mathrm{c}} / E_{0}\right)^{0.6} / 1+m_{2}\left(E_{\mathrm{c}} / E_{0}\right)^{0.6}
$$

with

and

$$
\begin{aligned}
& m_{1}=63.5 \log \left(0.0222 \Gamma_{\sigma} / A\right) \\
& m_{2}=0.54+25.9 A^{-0.25} \Gamma_{\sigma}^{-1} .
\end{aligned}
$$

The conduction model of Wu and Conrad gives

$$
\tau_{\mathrm{E}}=3 / 2 A_{\mathrm{s}} K_{\mathrm{f}} \phi \varepsilon_{0} E_{0}^{2} F \gamma /\left(1+\gamma^{2}\right)^{1 / 2}
$$

where

$$
F=66\left(\Gamma_{\sigma} / A\right)^{0.1}\left(E_{\mathrm{c}} / E_{0}\right)^{n}
$$

when $E_{\mathrm{c}}=0.1-0.3, n=1$ and the shear strain, $\gamma \sim 0.3$ at maximum attractive force between particles. The predicted values were calculated using eq 6 and 10 and compared with experimental values for the chitosan phosphate suspension.

The results are given in Figure 6. The experimental results were obtained at a shear rate of $2 \mathrm{~s}^{-1}$, volume fraction of 0.3 and electric fields of 1 to $3 \mathrm{kV} \mathrm{mm}^{-1}$. It 


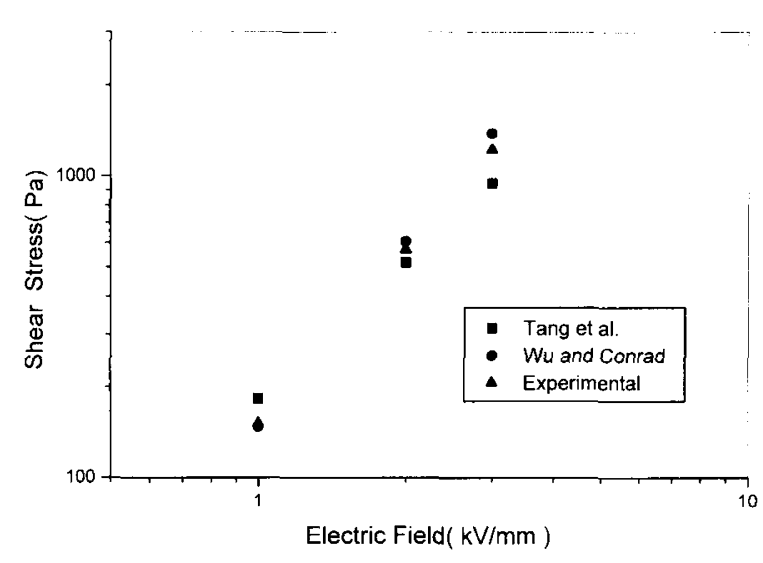

Figure 6. Comparision of predicted shear stress with that measured for chitosan phosphate suspension.

follows from eq 12 that at low shear rates the electric induced field, that is, the polarization force dominates, while at high shear rates the viscous shear force becomes dominant. The low shear rate regime has been termed quasi-static and the high shear rate regime dynamic. ${ }^{20}$ The results in Figures 4, 5, and 6 were obtained at a shear rate of $2 \mathrm{~s}^{-1}$ in the quasi-static regime, considerably less attention having been given to the more complex behavior in the dynamic regime

$$
\tau=\tau_{\mathrm{E}}+\tau_{\mathrm{V}}
$$

where $\tau_{\mathrm{E}}$ is the yield stress by electric induced field and $\tau_{\mathrm{V}}$ the flow stress at zero electric field. As seen in Figure 6 , the predicted values of all two conduction models are in accord with the experimental values.

\section{CONCLUSIONS}

This study investigates the electrorheological behavior of chitosan phosphate suspension and the following results were obtained:

(1) Chitosan phosphate suspension in silicone oil showed the ER response and exhibited an excellent shear yield stress upon the application of the electric field.

(2) Shear yield stress of the chitosan phosphate suspensions increased linearly with the volume fraction of the particles and the 1.67 power of the electric field.
(3) The values of structure factor, as obtained 3-4 for the chitosan phosphate suspension, possibly due to the formation of multiple chains upon application of the electric field.

(4) There is reasonable agreement between the predicted and experimental values of the shear yield stress for the chitosan phosphate suspension and the conduction models.

Acknowledgments. This research was sponsored by the NRL project of the Korean Ministry of Science and Technology.

\section{REFERENCES}

1. J. P. Shulman, R. G. Gorodkin, and E. V. Korobko, J. NonNewt. Fluid Mech., 8, 29 (1981).

2. K. D. Weiss and J. D. Carlson, J. Intell. Sys. and Struct., 4, 13 (1993).

3. H. Block and J. P. Kelly, J. Phys. D: Appl. Phys., 21, 1661 (1988).

4. D. J. Klingberg and C. F. Zukoski, Langmuir, 6, 15 (1990),

5. W. M. Winslow, J. Appl. Phys., 20, 1137 (1949).

6. A. P. Gast and C. F. Zukoski, Adv. Colloid Interface Sci., 30, 153 (1989).

7. T. C. Halsey and W. Toor, Phys. Rev. Lett., 65, 2820 (1990).

8. J. N. Foulc, N. Felici, and P. Atten, C. R. Acad. Sci. Paris., 314 II, 1279 (1992).

9. L. C. Davis and J. M. Ginder, "Progress in Electrorheology", K. O. Havelka and F. E. Filisko, Ed., Plenum Press, New York, N.Y., 1995, pp 107-114.

10. X. Tang, C. Wu, and H. Conrad, J. Rheol., 39, 1059 (1995).

11. C. Wu and H. Conrad, J. Phys. D: Appl. Phys., 29, 3147 (1996).

12. L. Onsager, J. Chem. Phys., 2, 599 (1934).

13. H. Uejima, Jpn. J. Appl. Phys., 11, 319 (1972).

14. H. Block and J. P. Kelly, Langmuir, 6, 6 (1990).

15. R. Bloodworth and E. Wendt, "Progress in Electrorheology", K. O. Havelka and F. E. Filisko, Ed., Plenum Press, New York, N.Y., 1995), pp 185-192.

16. C. J. Brine, "Advances in chitin and chitosan", C. J. Brine, P. A. Sandford, and J. P. Zikakis, Ed., Elsevier Applied Science, New York and London, 1992, pp 1-8.

17. U. S. Choi and H. Conrad, Int. Mec. Eng. Cong., Anaheim CA, USA., 1998.

18. T. Sakaguchi, T. Horikoshi, and A. Nakajima, Agric. Biol. Chem., 45, 2191 (1981).

19. H. Conrad, Y. Chen, and A. Sprecher, J. of Modn. Phys. B, 16, 2575 (1992).

20. H. Conrad and Y. Chen, "Progress in Electrorheology", K. O. Havelka and F. E. Filisko, Ed., Plenum Press, New York, N.Y., 1995, pp 55-85. 